\title{
On naked singularities in higher dimensional Vaidya space-times
}

\author{
S.G. Ghosh* \\ Department of Mathematics, Science College, Congress Nagar, \\ Nagpur-440 012, INDIA \\ and \\ Naresh Dadhich ${ }^{\dagger}$ \\ Inter-University Center for Astronomy and Astrophysics, \\ Post Bag 4, Ganeshkhind, Pune - 411 007, INDIA
}

\begin{abstract}
We investigate the end state of gravitational collapse of null fluid in higher dimensional space-times. Both naked singularities and black holes are shown to be developing as final outcome of the collapse. The naked singularity spectrum in collapsing Vaidya region $(4 D)$ gets covered with increase in dimensions and hence higher dimensions favor black hole in comparison to naked singularity. The Cosmic Censorship Conjecture will be fully respected for a space of infinite dimension.
\end{abstract}

PACS number(s): 04.20.Dw, 04.20.Cv, 04.70.Bw

\section{Introduction}

Inspired by work in string theory and other field theories, there has been a considerable interest in recent times to find solutions of the Einstein equation in dimensions greater than four. It is believed that underlying space-time in the large energy limit of the Planck energy may have higher dimensions than the usual four. At this level, all the basic forces of nature are supposed to unify and hence it would be pertinent in this context to consider solutions of the gravitational field equation in higher dimensions. Of course this consideration would be relevant when the usual four dimensional manifold picture of space-time becomes inapplicable. This would perhaps happen as we approach singularity whether in cosmology or in gravitational collapse.

Gravitational Collapse continues to occupy centre-stage in the gravitational research since the formulation of the singularity theorems [1] and cosmic censorship conjecture

\footnotetext{
*Author to whom all correspondence should be directed; email: sgghosh@hotmail.com
}

†email: nkd@iucaa.ernet.in 
(CCC) [2]. The singularity theorems revealed that the occurrence of singularities is a generic property of space-times in classical general relativity (GR). These theorems however say nothing about the detailed features of the singularities like their visibility to an external observer as well as their strength. On the other hand CCC states that GR contains a built in feature that precludes formation of naked singularities (see, [3], for reviews). The CCC remains as one of the most outstanding unresolved question in GR. However, there are many known examples in the literature showing that both naked singularities and black holes can form in gravitational collapse 4 .

The central shell focusing singularity can be naked or covered depending upon the choice of initial data. There is a critical branch of solution where a transition from naked singularity to black hole occurs. In particular gravitational collapse of spherical matter in the form of radiation (null fluid) described by Vaidya metric [5] is well studied for investigating CCC [6]-11].

The main aim of the paper is to examine what role dimensionality of space-time plays in the context of CCC. Interestingly it turns out that as dimension increases window for naked singularity shrinks. That is gravity seems to get strengthened with increase in dimensions of space. We shall first generalize previous studies of null fluid collapse to higher dimensional $(H D)$ space-times. The metric for the purpose is already known 12 and we shall call it $H D$ Vaidya metric. It turns out that higher dimensions seem to favor black hole. This would be discussed in the next section which would be followed by concluding discussion.

\section{Singularities in higher dimensional Vaidya space- times}

The metric of collapsing null fluid in $H D$ case is [12]

$$
d s^{2}=-\left[1-\frac{2 m(v)}{(n-1) r^{(n-1)}}\right] d v^{2}+2 d v d r+r^{2} d \Omega_{n}^{2}
$$

where $v \in(-\infty, \infty)$ is null coordinate which represents advanced Eddington time with, $r \in[0, \infty)$ is the radial coordinate,

$$
d \Omega_{n}^{2}=d \theta_{1}^{2}+\sin ^{2} \theta_{1}\left(d \theta_{2}^{2}+\sin ^{2} \theta_{2}^{2}\left(d \theta_{3}^{2}+\ldots \sin ^{2} \theta_{n}^{2}\right)\right.
$$

is a metric on $n$-sphere, and $n=D-2$, where $D$ is the total number of dimensions. The arbitrary function $m(v)$ (which is restricted only by the energy conditions), represents the mass at advanced time $v$. The energy momentum tensor can be written in the form

$$
T_{a b}=\frac{n}{(n-1) r^{n}} \dot{m}(v) k_{a} k_{b}
$$


with the null vector $k_{a}$ satisfying $k_{a}=-\delta_{a}^{v}$ and $k_{a} k^{a}=0$. We have used the units which fix the speed of light and gravitational constant via $8 \pi G=c=1$. Clearly, for the weak energy condition to be satisfied we require that $\dot{m}(v)$ to be non negative, where an over-dot represents a derivative with respect to $v$. Thus mass function is a non-negative increasing function of $v$ for imploding radiation.

\subsection{Self-similar case}

The physical situation here is that of a radial influx of null fluid in an initially empty region of the higher dimensional Minkowskian space-time. The first shell arrives at $r=0$ at time $v=0$ and the final at $v=T$. A central singularity of growing mass is developed at $r=0$. For $v<0$ we have $m(v)=0$, i.e., higher dimensional Minkowskian space-time, and for $v>T, \dot{m}(v)=0, m(v)$ is positive definite. The metric for $v=0$ to $v=T$ is $H D$ Vaidya, and for $v>T$ we have the $H D$ Schwarzschild solution. In order to get an analytical solution for our $H D$ case, we choose,

$$
2 m(v)= \begin{cases}0, & v<0 \\ \lambda(n-1) v^{(n-1)}(\lambda>0) & 0 \leq v \leq T \\ m_{0}(>0) & v>T\end{cases}
$$

With this choice of $m(v)$ the space-time is self similar [13], admitting a homothetic Killing vector which is given by Lie derivative

$$
\mathrm{E}_{\xi} g_{a b}=\xi_{a ; b}+\xi_{b ; a}=2 g_{a b}
$$

Let $K^{a}=d x^{a} / d k$ be the tangent vector to a null geodesic, where $k$ is an affine parameter. Along null geodesics, we have

$$
\xi^{a} K_{a}=r K_{r}+v K_{v}=C
$$

and writing $K^{v}=P / r$ as in [8], we obtain

$$
K^{r}=\left[1-\frac{2 m(v)}{(n-1) r^{(n-1)}}\right] \frac{P}{2 r} \text {. }
$$

Eq. (5), because of eqs. (3), $K^{v}$ and $K^{r}$, yields

$$
P=\frac{2 C}{2-\frac{v}{r}+\lambda\left(\frac{v}{r}\right)^{n}}
$$

and so the geodesics are completely determined. Radial null geodesics of the metric (11), by virtue of $K^{v}$ and $K^{r}$, satisfy

$$
\frac{d r}{d v}=\frac{1}{2}\left[1-\frac{2 m(v)}{(n-1) r^{(n-1)}}\right]
$$


Clearly, the above differential equation has a singularity at $r=0, v=0$. The nature (a naked singularity or a black hole) of the collapsing solutions can be characterized by the existence of radial null geodesics coming out of the singularity. The motion near singularity is characterized by the roots of an algebraic equation which we derive next. Eq. (8), upon using eq. (3), turns out to be

$$
\frac{d r}{d v}=\frac{1}{2}\left[1-\lambda X^{(n-1)}\right]
$$

where $X \equiv v / r$ is the tangent to a possible outgoing geodesic. The central shell focusing singularity is at least locally naked [14] (for brevity we have addressed it as naked throughout this paper), iff there exists $X_{0} \in(0, \infty)$ which satisfies

$$
X_{0}=\lim _{r \rightarrow 0 v \rightarrow 0} X=\lim _{r \rightarrow 0 v \rightarrow 0} \frac{v}{r}=\lim _{r \rightarrow 0} \frac{d v}{d r}=\frac{2}{1-\lambda X_{0}^{(n-1)}}
$$

or,

$$
\lambda X_{0}^{n}-X_{0}+2=0
$$

Thus any solution $X=X_{0}>0$ of the eq. (11) would correspond to naked singularity of the space-time, i.e., to future directed null geodesics emanating from the singularity $(v=0, r=0)$. The smallest such $X_{0}$ corresponds to the earliest ray emanating from the singularity and is called Cauchy horizon of the space-time. If $X_{0}$ is the smallest positive root of (11), then there are no naked singularities in the region $X<X_{0}$. Hence in the absence of positive real roots, the central singularity is not naked (censored) because in that case there are no outgoing future directed null geodesics from the singularity. Thus, occurrence of positive real roots implies that the strong CCC is violated, though not necessarily the weak CCC. The global nakedness of singularity can then be seen by making a junction onto $H D$ Schwarzschild space-time.

We now examine the condition for occurrence of naked singularity. With a straight forward calculation it can be shown that eq. (11) always admits two real positive roots for $\lambda \leq \lambda_{c}$, where $\lambda_{c}$ is the critical value of the parameter $\lambda$ deciding existence of naked singularity or black hole. The values of $\lambda_{c}$ and $X_{0}$ are summarized in the following two tables for the various $D$.

Table I: Variation of $\lambda_{c}$ and $X_{0}$ with $D$

\begin{tabular}{|c|l|l|}
\hline Dimensions $(D=n+2)$ & Critical value $\lambda_{c}=\frac{1}{n}\left(\frac{n-1}{2 n}\right)^{n-1}$ & Tangent $\left(X_{0}=\frac{2 n}{n-1}\right)$ \\
\hline 4 & $1 / 8$ & 4 \\
\hline 5 & $1 / 27$ & 3 \\
\hline 6 & $27 / 2048$ & 2.6667 \\
\hline 7 & $256 / 5000$ & 2.5 \\
\hline
\end{tabular}

Thus it follows that singularity will be naked if $\lambda \leq \lambda_{c}$. On the other hand if, the inequality is reversed, $\lambda>\lambda_{c}$ no naked singularity would form and gravitational collapse 
would result in black hole. Note that $X_{0}$ is bounded below by the value $2, X_{0} \rightarrow 2$ as $\lambda \rightarrow 0$ or $D \rightarrow \infty$.

Table II: Values of $X_{0}$ for $\lambda<\lambda_{c}$

\begin{tabular}{|c|l|l|}
\hline Dimensions $(D)$ & $\lambda<\lambda_{c}$ & Two Tangents $\left(X_{0}\right)$ \\
\hline 4 & .11 & $2.97086,6.12005$ \\
\hline 5 & .035 & $2.65512,3.49781$ \\
\hline 6 & .013 & $2.54764,2.80651$ \\
\hline 7 & .0051 & $2.45246,2.55146$ \\
\hline
\end{tabular}

It is interesting to note that $\lambda_{c}$ decreases significantly as we increase the value of $D$. Thus the spectrum of the naked singularity gets covered with introduction of extra dimensions (see Table I). The two roots in Table II indicate the naked singularity window in the slope of tangent to geodesics emanating from the singularity, which pinches with increase in dimension.

The degree of inhomogeneity of collapse is defined as $\mu \equiv 1 / \lambda$ (see [10]). Thus the inhomogeneity factor increases with D. From the physical point of view increase in inhomogeneity should favor naked singularity and hence should increase the spectrum. On the other hand increase in dimensions also strengthens gravity, which would go as $r^{(2-D)}$, as the collapse approaches singularity $r=0$. Amongst these two trends, the latter seems ultimately to have upper hand which results in shrinking of the naked singularity window for initial data.

Strength of the singularity: The strength of singularity, which is the measure of its destructive capacity, is the most important feature. Following Clark and Królak [15] we consider the null geodesics affinely parameterized by $k$ and terminating at shell focusing singularity $r=v=k=0$. Then it would be a strong curvature singularity as defined by Tipler [16] if

$$
\lim _{k \rightarrow 0} k^{2} \psi=\lim _{k \rightarrow 0} k^{2} R_{a b} K^{a} K^{b}>0
$$

where $R_{a b}$ is the Ricci tensor. It is widely believed that a space-time does not admit analytic extension through a singularity, if it is a strong curvature singularity in the above sense.

Eq. (12), with the help of eqs. (2), (3) and expression for $K^{v}$, can be expressed as

$$
\lim _{k \rightarrow 0} k^{2} \psi=\lim _{k \rightarrow 0} n \lambda X^{n-2}\left[\frac{k P}{r^{2}}\right]^{2} .
$$

Our purpose here is to investigate the above condition along future directed null geodesics coming out of the singularity. First, we note that

$$
\frac{d X}{d k}=\frac{1}{r} K^{v}-\frac{X}{r} K^{r}=\left(2-X-\lambda X^{n}\right) \frac{P}{2 r^{2}}=\frac{C}{r^{2}} .
$$


Using the fact that as singularity is approached, $k \rightarrow 0, r \rightarrow 0$ and $X \rightarrow a_{+}$(a root of (11)) and using L'Hôpital's rule, we observe

$$
\lim _{k \rightarrow 0} \frac{k P}{r^{2}}=\frac{2}{1+(n-2) \lambda X_{0}^{(n-1)}}
$$

and hence eq. (13) gives

$$
\lim _{k \rightarrow 0} k^{2} \psi=\frac{4 n \lambda X_{0}^{(n-2)}}{\left[1+(n-2) \lambda X_{0}^{(n-1)}\right]^{2}}>0 .
$$

Thus along the radial null geodesics strong curvature condition is satisfied and hence it is a strong curvature singularity.

\section{$2.2 \quad$ Non-self similar case}

In the previous section we have shown occurrence of strong curvature naked singularities for the self-similar $H D$ Vaidya space-times. Self-similarity is a strong geometric condition on the space-time. It may be argued that naked singularity could be an artifact of the self-similarity. It is therefore important to investigate non self-similar case as well. It has been shown that in $4 D$ naked-singularity does occur for non self-similar space-times [3, 8, 7]. In this section we wish to study the similar situation in $H D$ Vaidya space-times.

Here we examine the mass function given by

$$
m(v)=(n-1) \beta^{(n-1)} v^{\alpha(n-1)}\left[1-2 \alpha \beta v^{(\alpha-1)}\right]
$$

, $\alpha>1$ and $\beta$ are constants. This breaks the basic requirement for self-similarity [13]. This class of solutions for $4 D$ space-time have been discussed in [0, 9]. As mentioned above the null radiation shells start imploding at $v=0$ and the final shell arrives at $v=T$. The weak energy condition would require

$$
T^{\alpha-1}<\frac{n-1}{2 \beta(n \alpha-1)} .
$$

It is clear that $v=0, m(v)=0$, i.e., we shall have $H D$ Minkowskian and for $v=T$, $d m / d v=0$ and $m(v)=m_{0}(T)>0 H D$ Schwarzschild. The radial null geodesics for the mass function (17) can be obtained from eq. (8) and is given by $r=\beta v^{\alpha}$. This integral curve meets the singularity with a tangent at $r=0$ indicating occurrence of naked singularity. The singularity is also globally naked as $d r / d v>0$, with $v$ increasing. It is straight forward to see that $(n-1) r^{(n-1)}>2 m(T)$ is satisfied along this curve. Finally coming to the question of the strength of the singularity. It is seen that singularities are strong curvature only if $m(v) \sim v^{(n-1)}$ in the approach to singularity. 


\section{Concluding remarks}

In the absence of rigorous formulation as well as proof for either version of CCC, considerations of various examples showing occurrence of naked singularities remain the only tool to study this important problem. In this context, one question which could naturally arise is, what happens in higher dimensions which are currently being considered in view of their relevance for string theory and other field theories? Would the examples of naked singularity in $4 D$ go over to $H D$ or not? Our investigation shows that qualitatively the situation remains similar with monotonic shrinkage of naked singularity window with increase in dimensions. Increase in dimensions favors black hole. Our main aim was to study the effect of increase in dimension of the space on the collapse. As $D$ increases, two opposing effects set in, one increase in inhomogeneity and the other strengthening of gravitational field. The former would favour naked singularity while the latter black hole. It turns out that in the final analysis it is the latter that has an upper hand and leads to shrinkage of the naked singularity window. We have employed the Vaidya null radiation collapse scenario to study this effect.

Clearly the motivation for higher dimensions comes from the string theory in which the effective action involves the dilaton scalar field or anti-symmetric tensor field. The dilaton field couples non-minimally to the Ricci curvature. It would however be trivial in our case as the scalar curvature $R$ vanishes for the Vaidya solution. Similar would be the case for the anti-symmetric tensor field as well. Thus the results obtained here would also be relevant and valid for effective supergravity theories. It may also be noted that the higher curvature terms in the string theory become important only in the close vicinity of naked singularity when curvatures become divergingly high. That is when singularity is strong curvature singularity. That means our analysis of gravitational collapse would be relevant and meaningful for the effective SUGRA theories following from the string theory.

Vaidya metric in $4 D$ case has been extensively used to study the formation of naked singularity in spherical gravitational collapse. In this work we have generalized previous studies to the case of $H D$ Vaidya space-times. We have shown that results of gravitational collapse obtained in $4 D$ Vaidya space-time also go over to $H D$ Vaidya space-times and essentially retaining their physical behavior, i.e., strong curvature naked singularity.

Acknowledgment: SGG would like to thank IUCAA, Pune for the hospitality and UGC, Pune for MRP F. No. 23-118/2000 (WRO). The authors are grateful to anonymous referees for constructive criticism and suggestions.

\section{References}

[1] S. W. Hawking and G. F. R. Ellis, The Large Scale Structure of Space-time (Cambridge University Press, Cambridge, 1973). 
[2] R. Penrose, Riv. del Nuovo Cim. 1, 252 (1969); ibid. in General Relativity, an Einstein Centenary Volume, edited by S. W. Hawking and W. Israel (Cambridge University Press, England, 1979).

[3] P. S. Joshi, Global Aspects in Gravitation and Cosmology (Clendron Press, Oxford, 1993); C. J. S. Clarke, Class. Quantum Grav. 10, 1375 (1993); R. M. Wald, Preprint gr-qc/9710068 (1997); T. P. Singh, J. Astrophys. Astr. 20, 221 (1999). S. Jhingan and G. Magli, Preprint gr-qc/9903103 (1999); P. S. Joshi, Preprint gr-qc/0006101 (2000).

[4] See, for example, Joshi (Ref. [3],) (1993).

[5] P. C. Vaidya, Proc. Indian Acad. Sci. A33, 264 (1951); Reprinted, Gen. Rel. Grav. 31, 119 (1999).

[6] A. Papapetrou, in A Random Walk in Relativity and cosmology, edited by N. Dadhich, J. K. Rao, J. V. Narlikar and C. V. Vishveshwara, (Wiley, New York, 1985).

[7] K. Rajagopal and K. Lake Phys. Rev. D 35, 1531 (1987); K. Lake Phys. Rev. D 43, 1416 (1991).

[8] I. H. Dwivedi and P. S. Joshi, Class. Quantum Grav. 6, 1599 (1989); ibid. 8, 1339 (1991).

[9] P. S. Joshi and I. H. Dwivedi, J. Math. Phys. 32, 2167 (1991); ibid. Gen. Rel. Grav. 24, 129 (1992).

[10] J. P. S. Lemos, Phys. Rev. Lett. 68, 1447 (1992); ibid., Phys. Rev. D 59, 044020 (1992).

[11] S. Jhingan, N. Dadhich and P. S. Joshi, Preprint, gr-qc/0010111 (2000).

[12] B. R. Iyer and C. V. Vishveshwara, Pramana-J. Phys. 32, 749 (1989); L. K. Patel and N. Dadhich, gr-qc/9909068.

[13] A spherical symmetric space-time is self similar if $g_{t t}(c t, c r)=g_{t t}(t, r)$ and $g_{r r}(c t, c r)=$ $g_{r r}(t, r)$ for every $c>0$.

[14] Globally or locally naked singularities here means violating the weak or strong form of the CCC. Discussion of the two forms of CCC can be found in Ref. [2] (above).

[15] C. J. S. Clarke and A. Królak, J. Geom. Phys. 2, 127 (1986). 
[16] F. J. Tipler, C. J. S. Clarke, and G. F. R. Ellis, in General Relativity and Gravitation, edited by A. Held (Plenum, New York, 1980). 